\title{
Evaluating the association of common $L M N A$ variants with type 2 diabetes and quantitative metabolic phenotypes in French Europids
}

\author{
K. Duesing • G. Charpentier • M. Marre • J. Tichet • \\ S. Hercberg $\cdot$ P. Froguel $\cdot$ F. Gibson
}

Received: 4 September 2007 / Accepted: 27 September 2007 / Published online: 10 November 2007

(C) Springer-Verlag 2007

\begin{abstract}
Aims/hypothesis In the present study, we sought to examine the evidence that $L M N A$ variants are associated with type 2 diabetes and quantitative metabolic traits in French Europid individuals.

Methods We genotyped 24 single nucleotide polymorphisms (SNPs) spanning the LMNA gene in 3,093 case-control
\end{abstract}

Electronic supplementary material The online version of this article (doi:10.1007/s00125-007-0857-z) contains supplementary material, which is available to authorised users.

\author{
K. Duesing $\cdot$ P. Froguel $\cdot$ F. Gibson $(\bowtie)$ \\ Genomic Medicine, Imperial College, \\ Hammersmith Campus, Du Cane Road, \\ London W12 0NN, UK \\ e-mail: fernando.gibson@imperial.ac.uk \\ G. Charpentier \\ Endocrinology-Diabetology Unit, Corbeil Hospital, \\ Corbeil, France
}

M. Marre

Endocrinology-Diabetology, Bichat Hospital,

Paris, France

M. Marre

INSERM U695,

Paris, France

J. Tichet

Institut Régional Pour la Santé,

Tours, France

\section{S. Hercberg}

Scientific and Technical Institute of Nutrition and Food (ISTNA-CNAM), INSERM U557, INRA U1125,

Paris, France

P. Froguel

CNRS 8090, Institut de Biologie de Lille, Institut Pasteur,

CHU Lille, France participants. The association between LMNA SNPs and quantitative metabolic traits was also examined in the 1,674 normoglycaemic adults who made up the control cohort. Results SNP rs505058, a synonymous SNP (D446D) in exon 7, showed nominal evidence of association with type 2 diabetes $[p=0.003$, odds ratio (OR) 1.30 (95\% CI 1.09 1.56)] in French Europids. A meta-analysis of available rs505058 genotype data from 7,819 participants provided support for a modest association of rs505058 with type 2 diabetes $[p=0.003$, OR $1.19(95 \%$ CI $1.06-1.35)]$. We found no evidence $(p=0.91)$ that the tag SNP rs4641 is associated with type 2 diabetes. However, a meta-analysis of all available rs4641 genotype data in a total of 15,591 participants produced borderline evidence of association [ $p=0.054$, OR 1.05 (95\% CI 1.00-1.11)]. SNP rs6669212, in the $3^{\prime}$ untranslated region of $L M N A$, exhibited suggestive associations with WHR $(p=0.013)$, fasting serum levels of total cholesterol $(p=0.023)$ and triacylglycerol $(p=0.015)$. We emphasise that these quantitative trait associations are not corrected for multiple testing.

Conclusions/interpretation The available data do not support a major effect of common LMNA variation on type 2 diabetes susceptibility in northern Europeans. Further largescale studies are required to conclusively establish the extent to which LMNA variants have an impact on quantitative metabolic traits.

Keywords Case-control - Genetic association - LMNA . SNP. Tag SNP. Type 2 diabetes

$\begin{array}{ll}\text { Abbreviations } \\ \text { LD } & \text { linkage disequilibrium } \\ \text { MAF } & \text { minor allele frequency } \\ \text { OR } & \text { odds ratio } \\ \text { SNP } & \text { single nucleotide polymorphism }\end{array}$




\section{Introduction}

The LMNA gene is a biological and positional candidate susceptibility gene for type 2 diabetes. LMNA mutations cause profound insulin resistance and type 2 diabetes through the aetiology of familial partial lipodystrophy [1]. Positionally, the LMNA locus lies within the much-studied type 2 diabetes linkage region at chromosome 1q21-q24 [2].

Initial efforts to identify $L M N A$ variants associated with type 2 diabetes focused on the single nucleotide polymorphism (SNP) rs4641, a silent coding polymorphism $(\mathrm{H} 566 \mathrm{H})$ in exon 10 that is adjacent to the lamin $\mathrm{A} / \mathrm{C}$ alternative splice site. An association study of rs4641 carried out with participants recruited from the Pima Indian population found no association with type 2 diabetes [3]. A more comprehensive study involving the evaluation of six LMNA SNPs (including rs4641) in the Amish population also found no association with type 2 diabetes, but reported that rs4641 was associated with the metabolic syndrome and triacylglycerol levels [4]. Three well-powered studies have recently been published, each designed to examine the association of common variation spanning the LMNA gene with type 2 diabetes and lipodystrophy-related traits. The first of these [5] genotyped eight $L M N A$ tag SNPs in a large sample of Danish Europids (1,324 type 2 diabetes cases and 4,386 control participants) and found a modest association of rs4641 with type 2 diabetes, as well as nominally significant associations for other LMNA variants with quantitative metabolic and anthropometric traits. The second [6] found that none of the 13 LMNA SNPs tested was associated with type 2 diabetes or the metabolic syndrome in three large UK cohorts. Finally, another UK study [7] genotyped tag SNPs capturing an estimated 90\% of the common variation, but were unable to find an association with type 2 diabetes in 2,490 diabetes patients and 2,556 control participants. However, these authors also presented International Type 2 Diabetes 1q Consortium data, which indicated nominal associations for LMNA SNPs rs693671 and rs505058 in a small sample of French individuals [7]. In the present study, we set out to corroborate these findings in a large study of common $L M N A$ variation and type 2 diabetes in French Europid individuals. In addition, we sought to examine the evidence that $L M N A$ variants are associated with quantitative metabolic and anthropometric traits in normoglycaemic French adults.

\section{Methods}

Case-control participants All participants were of French Europid ancestry. Individuals identified by Sladek et al. [8] to lie outside the HapMap European Europid ancestry cluster were excluded from the study. Type 2 diabetes case participants were known diabetes patients. Normoglycaemic control participants were selected to have a fasting blood glucose concentration $<6.1 \mathrm{mmol} / 1$ [9]. Case participants were composed of: (1) 372 probands from diabetes families [10], recruited in Lille; and (2) 1,083 patients with a family history of type 2 diabetes recruited at the Corbeil-Essonne Hospital. Control participants were composed of: (1) 353 normoglycaemic parents from type 2 diabetes families; (2) 543 participants from the SUVIMAX prospective populationbased cohort study [11]; and (3) 742 participants selected from the DESIR cohort, a large prospective study of insulin resistance in French participants [12]. In total, the casecontrol cohort comprised 1,455 type 2 diabetic participants (age $60 \pm 12$ years; BMI $29.0 \pm 6.0 \mathrm{~kg} / \mathrm{m}^{2}$; men/women

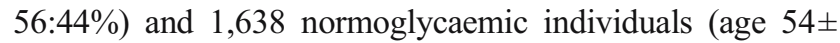
13 years; BMI $24.1 \pm 3.3 \mathrm{~kg} / \mathrm{m}^{2}$; men/women $43: 57 \%$ ). At $\alpha=0.05$, this sample size provides $94 \%$ power to detect a susceptibility allele with a frequency of 0.25 , assuming a disease prevalence of 0.1 and a heterozygote relative risk of 1.2 (multiplicative model) [13]. Informed consent was obtained from all participants and the study was approved by the local ethics committees.

SNP selection The LMNA gene locus extends for $25 \mathrm{~kb}$ $(154,351,122-154,376,495$ bp NCBI36) on chromosome 1q22. Our SNP selection strategy was based on providing dense genomic and functional coverage of the LMNA locus. The project was initiated before completion of the HapMap [14] and LMNA tag SNPs were added to the genotyped SNP set during the course of the genotyping. According to HapMap release \#21a, three tag SNPs (rs577492, rs582690 and rs4641) are sufficient to capture the common variation (minor allele frequency $[\mathrm{MAF}] \geq 5 \%$ ) across the locus in the Europid European population at $r^{2}>0.8$. The criteria for inclusion in the SNP set spanning the LMNA locus were as follows. (1) The three HapMap phase II tag SNPs (MAF $\geq 5 \% ; r^{2}=0.8$ ). (2) SNPs extracted from dbSNP and identified as residing in the following putative regulatory regions: the proximal promoter $(1 \mathrm{~kb}$ upstream of the RefSeq mRNA); exons plus 200 bp flanking intronic sequence; and conserved non-coding sequences (defined as $\geq 80 \%$ human-mouse identity across a $100 \mathrm{bp}$ window in the VISTA genome browser, http://pipeline.lbl.gov/cgi-bin/ gateway2). (3) Eight SNPs that showed preliminary association $(p<0.05)$ with type 2 diabetes in French samples of the 1q Consortium dataset ( 600 case-control samples).

SNP genotyping Genotyping was performed with the Sequenom MassARRAY system [15]. SNP genotype frequencies were tested for accordance with Hardy-Weinberg equilibrium using $\chi^{2}$ analysis. Quality control: SNPs with a call rate $<90 \%$, a MAF $<5 \%$, a Hardy-Weinberg $p<0.05$, or 
that exhibited poorly defined genotype clusters were disqualified from association analysis.

Statistical analyses To test for association of LMNA SNPs with type 2 diabetes, simple $\chi^{2}$ analysis of allelic and genotype counts was performed, with $p$ values presented uncorrected for multiple testing. To address the multiple testing problem, both the SNPspD method [16] and casecontrol data permutation using the Haploview software package [17] were employed. Combined analysis of association datasets and calculation of common odds ratios (ORs) was carried out with the Mantel-Haenszel metaanalysis method. Pairwise SNP linkage disequilibrium (LD) values and haplotype frequencies were calculated with Haploview. The quantitative anthropometrical (height, BMI, WHR) and metabolic (fasting serum levels of triacylglycerol, total cholesterol, glucose and insulin) phenotypes measured in the 1,674 normoglycaemic control participants were log transformed and adjusted for age, sex and BMI, as appropriate. Testing SNPs for association with quantitative traits was carried out with SPSS 14.0 using the ANOVA test and a codominant model. Quantitative trait association $p$ values are presented uncorrected for multiple testing. The Haplotype Trend Regression program [18] was used to test inferred haplotypes for association with quantitative traits.

\section{Results}

We tested a total of 24 SNPs spanning the LMNA gene for association with type 2 diabetes in 3,093 French participants. Genotype call rates exceeded $90 \%$ overall. The high density of the SNP set, which included three HapMap II tag SNPs, represented comprehensive coverage of the common variation at the LMNA locus. The minor allele of rs505058, a synonymous SNP (D446D) in exon 7, showed nominal evidence of association with type 2 diabetes $[p=0.003$,
OR 1.30 (95\% CI 1.09-1.56); Table 1 and Electronic supplementary material (ESM) Table 1]. Genotype counts for all SNPs are presented in ESM Table 2. The SNPSpD multiple testing correction method [16] gave a significance threshold of $p=0.007$ for the 24 SNPs tested, equivalent to seven independent tests at $\alpha=0.05$. Case-control permutation analysis of the rs505058 genotype data $(10,000$ permutations) produced a modest empirical $p$ value of 0.03 for the association result.

Given that the rs505058 association result represents an allele frequency difference of only $2 \%$ between the case and control groups, it was pertinent to examine the quality of the genotype data generated for this variant. The overlap between the samples in the present study and that of the 1q Consortium allowed us to estimate the genotyping error rate for rs505058. There were 476 samples (176 cases, 300 controls) with rs505058 genotypes in common in both datasets. All 476 genotypes were concordant between the two studies and genotyping chemistries (the 1q Consortium used an Illumina platform) equating to an error rate of $<0.2 \%$. Removing the overlapping 476 samples from the analysis reduced the association to that of a trend $(p=0.08)$. Combining our rs505058 data with those of the UK study of Owen et al. [7] reduced the allele frequency difference between the case and control groups to $1 \%$ but nevertheless provided support for a modest association of rs505058 with type 2 diabetes $[p=0.003$, OR 1.19 (95\% CI 1.06-1.35)] (Table 2).

We found no evidence $(p=0.91)$ that the tag SNP rs4641 is associated with type 2 diabetes in French Europids, in agreement with both of the UK studies [6, 7]. However, combining our data for rs 4641 with those of the three recently published $L M N A$ studies [5-7] produced borderline evidence of association for $\operatorname{rs} 4641[p=0.054$, OR 1.05 (95\% CI 1.00-1.11)] (Table 3). SNP rs693671, reported to be associated with type 2 diabetes in the French samples of the 1q Consortium dataset [7] was not corroborated in the larger sample size employed in the present study, confirming the negative result obtained for this SNP in the UK studies $[6,7]$.

Table 1 Association of LMNA SNPs rs505058 and rs4641 with type 2 diabetes in French Europids

\begin{tabular}{|c|c|c|c|c|c|c|c|c|c|}
\hline SNP & $\begin{array}{l}\text { SNP } \\
\text { feature }^{a}\end{array}$ & Allele $^{\mathrm{b}}$ & $\begin{array}{l}\text { Chr position } \\
\text { (NCBI36) }\end{array}$ & $\begin{array}{l}\text { Gene } \\
\text { region }\end{array}$ & Status & $\begin{array}{l}\text { No. } \\
\text { participants }\end{array}$ & $\begin{array}{l}\text { Allele } 1 \\
(\%)\end{array}$ & $\begin{array}{l}\text { Allele } 2 \\
(\%)\end{array}$ & $p$ value \\
\hline \multirow[t]{2}{*}{ rs 505058} & $1 \mathrm{qC}$ & $\mathrm{T} / \mathrm{C}$ & $154,372,809$ & Exon 7 & $\mathrm{~T} 2 \mathrm{D}$ & 1,426 & $2,556(90)$ & $296(10)$ & 0.003 \\
\hline & & & & & NG & 1,591 & $2,922(92)$ & $260(8)$ & \\
\hline \multirow[t]{2}{*}{ rs4641 } & Tag & $\mathrm{C} / \mathrm{T}$ & $154,374,158$ & Exon 10 & $\mathrm{~T} 2 \mathrm{D}$ & 1,408 & 2,079 (74) & $737(26)$ & 0.91 \\
\hline & & & & & NG & 1,595 & $2,351(74)$ & 839 (26) & \\
\hline
\end{tabular}

Association $p$ values were generated by $\chi^{2}$ analysis of the allelic counts.

${ }^{a} 1 \mathrm{qC}$ : SNP showed nominal evidence of association with type 2 diabetes $(p<0.05)$ in the French samples of the International Type 2 Diabetes $1 \mathrm{q}$ Consortium; Tag: HapMap phase II tag SNP.

${ }^{\mathrm{b}} \mathrm{SNP}$ alleles are shown as major/minor.

NG, normoglycaemic control participants; T2D, type 2 diabetes cases 
Table 2 Meta-analysis of the association of LMNA SNP rs505058 with type 2 diabetes

\begin{tabular}{|c|c|c|c|c|c|}
\hline Study & Allele $1(\%)$ & Allele $2(\%)$ & No. participants & $p$ value & OR $(95 \% \mathrm{CI})$ \\
\hline \multicolumn{6}{|l|}{ Present } \\
\hline $\mathrm{T} 2 \mathrm{D}$ & $2,556(90)$ & $296(10)$ & 1,426 & \multirow[t]{2}{*}{0.003} & \multirow[t]{2}{*}{$1.30(1.09-1.55)$} \\
\hline NG & $2,922(92)$ & $260(8)$ & 1,591 & & \\
\hline \multicolumn{6}{|c|}{ Owen et al. [7] } \\
\hline $\mathrm{T} 2 \mathrm{D}$ & 4,469 (93) & $329(7)$ & 2,399 & \multirow[t]{2}{*}{0.208} & \multirow[t]{2}{*}{$1.11(0.94-1.30)$} \\
\hline NG & $4,507(94)$ & $299(6)$ & 2,403 & & \\
\hline \multicolumn{6}{|c|}{ Combined data } \\
\hline T2D & $7,025(92)$ & $625(8)$ & 3,825 & \multirow[b]{2}{*}{0.003} & \multirow[b]{2}{*}{$1.19(1.06-1.35$} \\
\hline NG & $7,429(93)$ & $559(7)$ & 3,994 & & \\
\hline
\end{tabular}

Allelic count data taken from the present study and Owen et al. [7]. The combined OR was calculated with the Mantel-Haenszel method $\mathrm{NG}$, normoglycaemic control participants; T2D, type 2 diabetes cases

The LD pattern across LMNA shows two 'blocks' of LD (Fig. 1), in good agreement with the LD structure described in previous studies $[5,6]$. However, it is clear that the three HapMap tag SNPs (rs577492, rs582690 and rs4641) do not tag all the SNPs typed in the study, justifying our decision to type additional SNPs. The LD data provide support for the rs505058 result since the two SNPs that are proxies at $r^{2}>0.9$ for rs505058 (rs547915 and rs538089) showed weak nominal association with type 2 diabetes $(p=0.04)$. Common haplotypes from each block were analysed for association with type 2 diabetes. No haplotype was associated with type 2 diabetes (data not shown). There were no significant differences in SNP allele or haplotype frequencies between men and women, and no association with type 2 diabetes was uncovered by stratifying for sex (data not shown).

The 24 LMNA SNPs were also tested for association with a number of metabolic and anthropometrical quanti- tative phenotypes (ESM Table 3). SNP rs6669212, in the 3' untranslated region of $L M N A$, which had shown borderline association with type 2 diabetes, exhibited suggestive pleiotropic associations (Table 4) with WHR $(p=0.013)$, and fasting serum levels of total cholesterol $(p=0.023)$ and triacylglycerol $(p=0.015)$. In addition, SNP rs7339 in exon 12 was associated with height $(p=0.018)$. We emphasise that these quantitative trait associations are of nominal significance and therefore are not corrected for multiple testing. Haplotype analysis did not identify any additional associations with continuous traits (data not shown).

\section{Discussion}

We have carried out a comprehensive association study of common genetic variation spanning the LMNA locus and

Table 3 Meta-analysis of the association of LMNA SNP rs4641 with type 2 diabetes

\begin{tabular}{|c|c|c|c|c|c|}
\hline & Allele $1(\%)$ & Allele $2(\%)$ & No. participants & $p$ value & OR $(95 \% \mathrm{CI})$ \\
\hline \multicolumn{6}{|l|}{ Present } \\
\hline $\mathrm{T} 2 \mathrm{D}$ & 2,079 (74) & 737 (26) & 1,426 & \multirow[t]{2}{*}{0.910} & \multirow[t]{2}{*}{$0.99(0.89-1.12)$} \\
\hline NG & $2,351(74)$ & 839 (26) & 1,595 & & \\
\hline \multicolumn{6}{|c|}{ Owen et al. [7] } \\
\hline $\mathrm{T} 2 \mathrm{D}$ & $3,441(72)$ & $1,345(28)$ & 2,393 & \multirow[t]{2}{*}{0.148} & \multirow[t]{2}{*}{$1.07(0.98-1.17)$} \\
\hline NG & $3,580(73)$ & $1,310(27)$ & 2,445 & & \\
\hline \multicolumn{6}{|c|}{ Mesa et al. [6] } \\
\hline $\mathrm{T} 2 \mathrm{D}$ & $1,222(74)$ & $434(26)$ & 828 & \multirow[t]{2}{*}{0.507} & \multirow[t]{2}{*}{$0.95(0.83-1.10)$} \\
\hline $\mathrm{NG}$ & $1,766(73)$ & $658(27)$ & 1,212 & & \\
\hline \multicolumn{6}{|c|}{ Wegner et al. [5] } \\
\hline $\mathrm{T} 2 \mathrm{D}$ & $1,934(73)$ & $714(27)$ & 1,324 & \multirow[t]{2}{*}{0.010} & \multirow[t]{2}{*}{$1.14(1.03-1.26)$} \\
\hline $\mathrm{NG}$ & $6,623(76)$ & 2,149 (24) & 4,386 & & \\
\hline \multicolumn{6}{|c|}{ Combined data } \\
\hline $\mathrm{T} 2 \mathrm{D}$ & $8,676(73)$ & $3,230(27)$ & 5,953 & \multirow[t]{2}{*}{0.054} & \multirow[t]{2}{*}{$1.05(1.00-1.11)$} \\
\hline NG & $14,320(74)$ & $4,956(26)$ & 9,638 & & \\
\hline
\end{tabular}

Allelic count data from the present study, Wegner et al. [5], Mesa et al. [6] and Owen et al. [7]. The combined OR was calculated with the MantelHaenszel method.

NG, normoglycaemic control participants; T2D, type 2 diabetes cases 


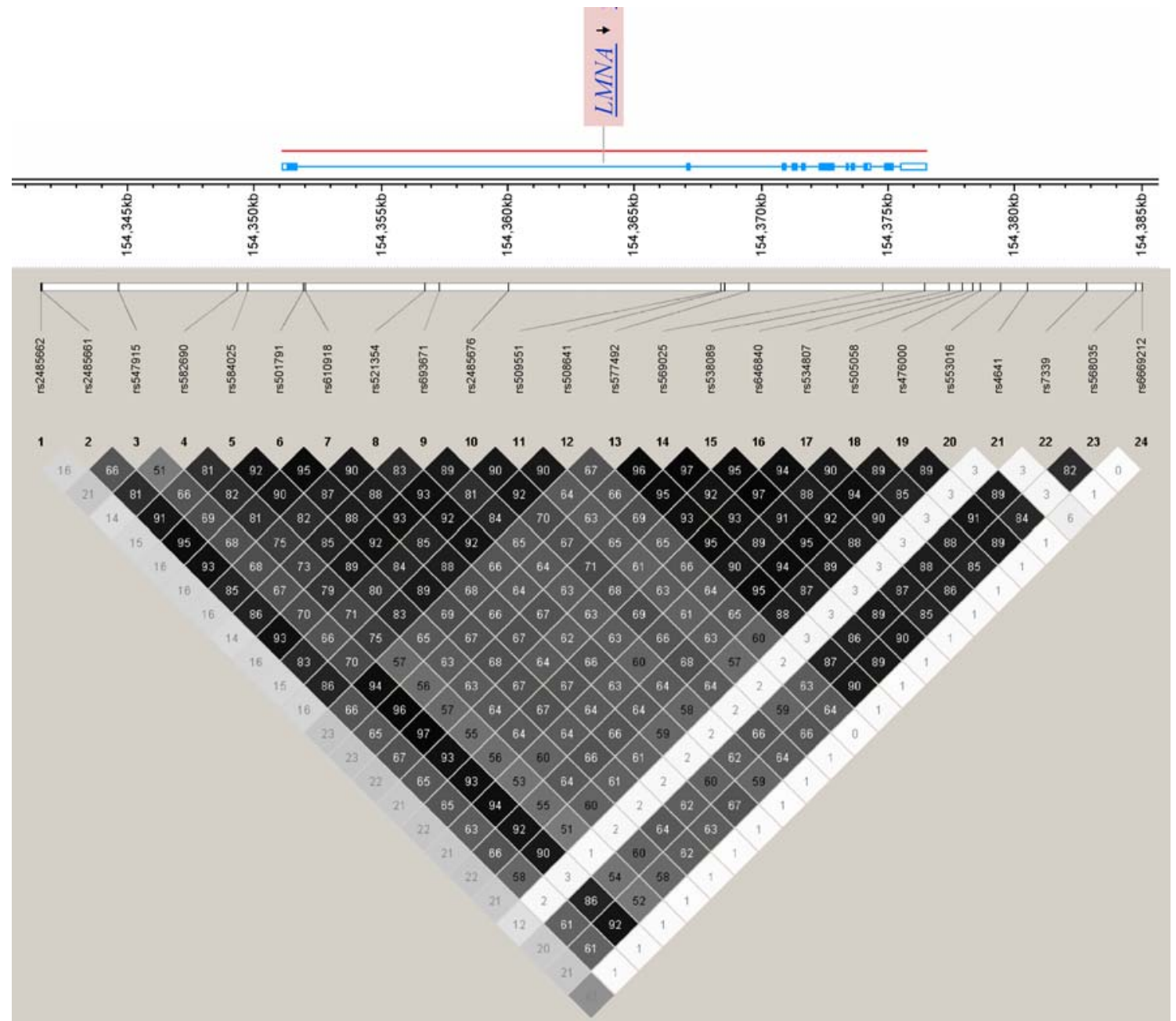

Fig. 1 Pattern of LD across the LMNA locus. Haploview plot of pairwise SNP $r^{2}$ values calculated from the genotype data of the control participant samples

type 2 diabetes. We obtained evidence supporting a modest association of the synonymous SNP rs505058 (D446D) with type 2 diabetes in French Europids, and a combined analysis of our data with those from a large UK study [7] provided support for this finding.

We found no evidence that the tag SNP rs4641 is associated with type 2 diabetes in French Europids; and a meta-analysis of all available published data for this SNP produced evidence of association that did not attain statistical significance. Thus, the available data do not support a major effect on type 2 diabetes susceptibility for rs4641 or indeed any other common $L M N A$ variant in northern Europeans.

We have also presented evidence that SNP rs6669212 exhibited pleiotropic associations with WHR, total cholesterol and triacylglycerol. Although three previous studies

Table $4 L M N A$ SNPs associated with quantitative metabolic and anthropometrical phenotypes

\begin{tabular}{|c|c|c|c|c|}
\hline & $1 / 1$ & $1 / 2$ & $2 / 2$ & $p$ value \\
\hline \multicolumn{5}{|l|}{ rs6669212 } \\
\hline WHR & $0.83 \pm 0.05(446)$ & $0.82 \pm 0.05(148)$ & $0.79 \pm 0.08(16)$ & 0.013 \\
\hline Total cholesterol $(\mathrm{mmol} / \mathrm{l})$ & $5.92 \pm 1.03(458)$ & $5.90 \pm 1.03(152)$ & $5.81 \pm 1.04(17)$ & 0.023 \\
\hline Triacylglycerol (mmol/l) & $0.98 \pm 1.21(455)$ & $0.97 \pm 1.21(152)$ & $0.86 \pm 1.27(17)$ & 0.015 \\
\hline \multicolumn{5}{|l|}{ rs7339 } \\
\hline Height (cm) & $165.2 \pm 1.0(1,086)$ & $165.1 \pm 1.0(210)$ & $166.1 \pm 1.0(16)$ & 0.018 \\
\hline
\end{tabular}

Data are means \pm SD

The number of participants in each genotype class are shown in parentheses. 
also identified associations of LMNA SNPs with quantitative traits $[5,19,20]$, our quantitative trait results must be interpreted with some caution for the following reasons. First, our findings do not represent replication of previous results - the SNPs associated with metabolic quantitative traits in this study are different from those identified previously. Second, the quantitative trait associations reported here are not particularly strong and therefore do not stand up to multiple testing correction. On the other hand, several independent studies have now found associations of LMNA SNPs with quantitative metabolic traits, findings that are consistent with the phenotype of both human lipodystrophy and the Lmna knockout mouse, which shows growth retardation and multiple metabolic abnormalities [21].

In conclusion, the available data do not support a major effect of common $L M N A$ variation on type 2 diabetes susceptibility in northern Europeans. Further detailed analysis, including meta-analyses of existing data or testing in additional large cohorts, is required to conclusively establish the extent to which LMNA variants have an impact on quantitative metabolic traits.

Acknowledgements This work was supported by a Wellcome Trust University Award to F. Gibson (GR065414MF) and a UK Medical Research Council Programme Grant to P. Froguel (G0000477). We thank the anonymous referees and editorial staff for critically appraising and improving draft versions of the manuscript.

Duality of interest The authors declare that there is no duality of interest associated with this manuscript.

\section{References}

1. Cao H, Hegele RA (2000) Nuclear lamin A/C R482Q mutation in Canadian kindreds with Dunnigan-type familial partial lipodystrophy. Hum Mol Genet 9:109-112

2. McCarthy MI (2003) Growing evidence for diabetes susceptibility genes from genome scan data. Curr Diab Rep 3:159-167

3. Wolford JK, Hanson RL, Bogardus C, Prochazka M (2001) Analysis of the lamin A/C gene as a candidate for type II diabetes susceptibility in Pima Indians. Diabetologia 44:779-782

4. Steinle NI, Kazlauskaite R, Imumorin IG et al (2004) Variation in the lamin A/C gene: associations with metabolic syndrome. Arterioscler Thromb Vasc Biol 24:1708-1713

5. Wegner L, Andersen G, Sparso T et al (2007) Common variation in LMNA Increases susceptibility to type 2 diabetes and associates with elevated fasting glycemia and estimates of body fat and height in the general population: studies of 7,495 Danish whites. Diabetes 56:694-698

6. Mesa JL, Loos RJ, Franks PW et al (2007) Lamin a/c polymorphisms, type 2 diabetes, and the metabolic syndrome: casecontrol and quantitative trait studies. Diabetes 56:884-889

7. Owen KR, Groves CJ, Hanson RL et al (2007) Common variation in the LMNA Gene (encoding lamin $\mathrm{A} / \mathrm{C}$ ) and type 2 diabetes: association analyses in 9,518 subjects. Diabetes 56:879-883

8. Sladek R, Rocheleau G, Rung J et al (2007) A genome-wide association study identifies novel risk loci for type 2 diabetes. Nature 445:881-885

9. Report of the expert committee on the diagnosis and classification of diabetes mellitus (2003) Diabetes Care 26(Suppl 1):S5-S20

10. Vionnet N, Hani EH, Dupont S et al (2000) Genomewide search for type 2 diabetes-susceptibility genes in French whites: evidence for a novel susceptibility locus for early-onset diabetes on chromosome 3q27-qter and independent replication of a type 2diabetes locus on chromosome 1q21-q24. Am J Hum Genet 67:1470-1480

11. Hercberg S, Preziosi P, Briancon S et al (1998) A primary prevention trial using nutritional doses of antioxidant vitamins and minerals in cardiovascular diseases and cancers in a general population: the SU.VI.MAX study - design, methods, and participant characteristics. SUpplementation en VItamines et Mineraux AntioXydants. Control Clin Trials 19:336-351

12. Balkau B (1996) An epidemiologic survey from a network of French Health Examination Centres (D.E.S.I.R.): epidemiologic data on the insulin resistance syndrome. Rev Epidemiol Sante Publique 44:373-375 (in French)

13. Purcell S, Cherny SS, Sham PC (2003) Genetic power calculator: design of linkage and association genetic mapping studies of complex traits. Bioinformatics 19:149-150

14. The International HapMap Consortium (2005) A haplotype map of the human genome. Nature 437:1299-1320

15. Jurinke C, van den Boom D, Cantor CR, Koster H (2002) Automated genotyping using the DNA MassArray technology. Methods Mol Biol 187:179-192

16. Nyholt DR (2004) A simple correction for multiple testing for single-nucleotide polymorphisms in linkage disequilibrium with each other. Am J Hum Genet 74:765-769

17. Barrett JC, Fry B, Maller J, Daly MJ (2005) Haploview: analysis and visualization of $\mathrm{LD}$ and haplotype maps. Bioinformatics 21:263-265

18. Zaykin DV, Westfall PH, Young SS, Karnoub MA, Wagner MJ, Ehm MG (2002) Testing association of statistically inferred haplotypes with discrete and continuous traits in samples of unrelated individuals. Hum Hered 53:79-91

19. Hegele RA, Cao H, Harris SB, Zinman B, Hanley AJ, Anderson CM (2000) Genetic variation in LMNA modulates plasma leptin and indices of obesity in aboriginal Canadians. Physiol Genomics 3:39-44

20. Hegele RA, Huff MW, Young TK (2001) Common genomic variation in LMNA modulates indexes of obesity in Inuit. J Clinical Endocrinol Metab 86:2747-2751

21. Sullivan T, Escalante-Alcalde D, Bhatt H et al (1999) Loss of A-type lamin expression compromises nuclear envelope integrity leading to muscular dystrophy. J Cell Biol 147:913-920 